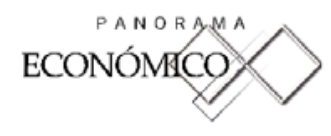

Volumen VII, No. 13

Julio-Diciembre, 2011, pp. 23-52

\title{
LOS EFECTOS DE LA POLÍTICA FISCAL Y EL PRODUCTO EXTERNO EN UNA ECONOMÍA EMERGENTE ABIERTA AL EXTERIOR
}

\author{
Alejandro Rodríguez Arana*
}

(Recibido: Febrero 2011 / Aprobado: Mayo 2011)

\begin{abstract}
Resumen
En corto plazo los efectos de una política fiscal expansiva y de un incremento en el producto externo son similares para el producto doméstico y el tipo de cambio real. Sin embargo, en el largo plazo la política fiscal expansiva provoca una reducción neta del producto doméstico y un incremento neto del tipo de cambio real, mientras que el aumento del producto externo incrementa aún más el producto doméstico y propicia una reducción adicional del tipo de cambio real. Ante una caída del producto externo, una política fiscal contracíclica puede estabilizar el producto doméstico en corto plazo. En largo plazo se refuerzan los efectos recesivos del choque original.

Palabras clave: política fiscal, cuenta corriente, producto externo, tipo de cambio real
\end{abstract}

* Universidad Iberoamericana, Plantel Ciudad de México. Correo electrónico: <alejandro.rodriguez@uia.mx>. 


\begin{abstract}
The effects of an expansive fiscal policy and an increase in foreign output are similar for the domestic output and the real exchange rate in the short run. However, in the long run an expansive fiscal policy generates a net reduction of the domestic output and a net increase of the real exchange rate, while the increase in foreign output increases even more the domestic output and generates an additional reduction of the real exchange rate. When foreign output falls, a countercyclical fiscal policy may stabilize the domestic output in the short run. In the long run, however, this policy reinforces the recessive effects of the original shock.
\end{abstract}

Keywords: fiscal policy, current account, foreign output, real exchange rate

Clasificación JEL: E32, E62, F32, F41

\title{
Introducción
}

La actividad productiva de diversas economías emergentes está fuertemente relacionada con la correspondiente en economías desarrolladas. Entre 1996 y 2010 la correlación lineal entre el componente cíclico del PIB en México y el correspondiente a Estados Unidos fue de 0.92 . Cuando se toma el crecimiento de ambas economías, la misma medida es de casi $90 \%$. La interacción entre estas dos economías propició que la recesión de Estados Unidos de 2009, donde el producto cayó en 2.7\%, diera lugar a una caída mucho más pronunciada en el producto de México (de 6.2\%).

Un choque externo negativo en una economía emergente cuestiona seriamente el papel de la política económica. Si se utilizan políticas expansivas ¿qué tanto es posible suavizar el choque? ¿Debería utilizarse una política fiscal o una política monetaria? ¿O sería tal vez mejor no llevar a cabo política alguna?

Este trabajo está motivado en la observación de la relación de México y Estados Unidos y, considerando ésta como punto de partida, hace un análisis a nivel teórico válido para otras posibles relaciones entre economías emergentes y desarrolladas en otros lugares del mundo.

Un enorme obstáculo al analizar este tipo de problemas es el metodológico. Antes de la crisis financiera de 2007-2009 en el mundo desarrollado, la macroeconomía había llegado a un cierto consenso (ver Blanchard, 2008; Woodford, 2008). Dicho 
consenso une la teoría de los nuevos keynesianos (the new keynesian theory) con la teoría del ciclo real (real business cycle). En esta óptica los ciclos económicos ocurren tanto por movimientos tecnológicos como por cambios en la demanda en presencia de precios rígidos. ${ }^{1}$

El consenso supone mercados completos y consumidores y productores que optimizan utilidad y ganancias. La única fricción es la de precios predeterminados que se ajustan de manera lenta. En este tipo de modelos generalmente tienen lugar dos resultados: la política fiscal es irrelevante, frecuentemente en todo momento, y el producto de largo plazo está determinado por una oferta fija de factores de producción.

Las observaciones empíricas que señalamos en párrafos anteriores sobre la relación del producto de México con el de Estados Unidos, pero también en el papel de la política fiscal, parecen contradecir los resultados generales del consenso. A fines de los años setenta y principios de los ochenta en México y otros países, como Argentina y Uruguay, una política fiscal expansiva propició un fuerte incremento del producto y del consumo, así como un enorme déficit de la cuenta corriente. Después, estos países experimentaron crisis en los que el producto cayó notablemente.

Varios trabajos empíricos muestran una relación positiva entre el déficit fiscal y el déficit en cuenta corriente en diversos países (ver por ejemplo Bernheim, 1987; Funke y Nickel, 2006; Nickel y Vansteenkiste, 2008). ${ }^{2}$ En Estados Unidos esta relación se conoce como "de los déficit gemelos" (twin deficits). El análisis de las reversiones en el déficit en cuenta corriente muestra que en tales situaciones normalmente ocurren fuertes recesiones posteriores (ver Millesi-Ferreti y Razin, 1998; Edwards, 2004).

En el terreno de la metodología, la crisis financiera del mundo desarrollado ha ocasionado una airada discusión en el área macroeconómica. Krugman (2009) y Buiter (2009) critican, tal vez de manera demasiado informal, las premisas del ya mencionado consenso entre la teoría de los nuevos keynesianos y el ciclo real. Gordon (2009) hace un análisis más balanceado y propone, concretamente, volver al modelo básico propuesto por él mismo (Gordon, 1978) y por Dornbusch y Fischer (1978), el cual parte, por el lado de la demanda, de un esquema muy similar al del modelo

El consenso señalado por Blanchard (2008) y Woodford (2008) probablemente muestre una visión optimista de estos autores. Difícilmente los creadores de la teoría del ciclo real (Kydland y Prescott) verían posible dicho consenso.

2 Para una versión escéptica ver Kim y Roubini (2004). 
IS-LM, y por el lado de la oferta de una curva de Phillips que considera inercia tanto en el sistema de precios como en la inflación misma. Siguiendo una línea similar a la de Gordon (2009), Drifill y Miller (2009) proponen analizar problemas como restricciones de liquidez y de los mercados de crédito, lo cual lleva a una versión moderna de la demanda agregada también similar a la del modelo IS-LM tradicional.

El presente trabajo lleva a cabo un ejercicio para destacar los efectos de la política fiscal y del producto externo sobre la actividad económica y otras variables de una economía emergente. Siguiendo la línea de Gordon (2009), el artículo utiliza un esquema que podría denominarse keynesiano, pues toma en cuenta a la muy denostada función IS como elemento fundamental de la demanda agregada. Sin embargo, el análisis es de equilibrio pues la demanda agregada es en todo momento igual a la oferta agregada. ${ }^{3}$

Una razón de peso para modelar la demanda a partir de la antigua función IS es que, de acuerdo a diversos trabajos empíricos, el ingreso corriente tiene un efecto muy importante en el consumo. Cambpell y Mankiw (1990) encuentran que en Estados Unidos - posiblemente el país con un mercado de capitales más desarrollado del mundo- el ingreso corriente determina cuando menos el 50\% del consumo. En economías emergentes este efecto podría ser mucho mayor e invalidaría la llamada ecuación de Euler, o de Ramsey-Keynes, de sustitución intertemporal del consumo, la cual ha sustituido a la antigua IS en muchos modelos macroeconómicos modernos (ver Blanchard, 2008).

El artículo muestra que en el corto plazo una política fiscal expansiva y un incremento del producto externo tienen efectos similares sobre el producto doméstico, el cual aumenta, y el tipo de cambio real, el cual baja. Sin embargo, la política fiscal deteriora la cuenta corriente, mientras que el incremento del producto externo la mejora.

Los resultados opuestos sobre la cuenta corriente de la política fiscal expansiva y el incremento del producto externo propician efectos diversos de largo plazo. Al final, una política fiscal expansiva termina reduciendo en términos netos el producto

\footnotetext{
Este trabajo toma la visión de Tobin (1993) de que la diferencia entre una economía keynesiana y una clásica no tiene relación con la rigidez de precios sino más bien con la estructura de la demanda, la cual en un mundo keynesiano tiene un grado de independencia importante dado por los componentes autónomos del consumo y la inversión. Bajo esta óptica, los modelos llamados de la nueva economía keynesiana no son realmente keynesianos, son modelos con una estructura clásica pero que muestran rigidez en los precios. En cambio puede haber modelos que presentan una estructura keynesiana, como el aquí presentado, pero donde los precios relativos son absolutamente flexibles.
} 
doméstico e incrementando el tipo de cambio real. En cambio, el aumento del producto externo propicia un incremento del producto doméstico todavía mayor y una reducción adicional del tipo de cambio real.

El corolario de estos hallazgos es que ante un choque de demanda negativo provocado por una reducción del producto externo, una política fiscal contracíclica puede estabilizar el producto en el corto plazo. No obstante, llevarla a cabo traerá efectos de largo plazo altamente recesivos, pues dicha política fiscal atizará sobre el déficit de cuenta corriente en el corto plazo.

El artículo está dividido en dos secciones: la primera muestra el modelo de cortomediano plazo y los efectos iniciales de una política fiscal expansiva, o de un incremento del producto externo, sobre el producto doméstico, el tipo de cambio real y la balanza comercial; la segunda parte lleva a cabo una extensión que incorpora el comportamiento de la cuenta corriente y de los activos netos del exterior sobre la demanda agregada. Aquí se indaga el efecto de largo plazo de una caída en el producto externo y una política fiscal contracíclica.

\section{Un modelo dinámico de economía emergente abierta al exterior}

En una línea similar a la de Layard, Nickell y Jackman (1992, capítulo 8) y Harrigan et al. (1993), el contexto que se presenta es el de una economía emergente abierta al exterior. Este tipo de economías son pequeñas en el sentido de que su producción no puede influir a la producción de las economías desarrolladas. No obstante, la producción de la economía emergente presenta un cierto grado de diferenciación y, por lo tanto, el precio de los productos domésticos comerciables está determinado en forma endógena.

En contraste, en una economía realmente pequeña, la producción de esa entidad no puede influir la producción de las economías grandes, pero además el precio de los bienes comerciables que dicha economía produce está determinado totalmente en los mercados internacionales.

Para el caso de economías emergentes que nos ocupa, el análisis hace abstracción de la producción de bienes no comerciables y supone que todos los bienes son comerciables. La relación de los precios externos en moneda doméstica divididos por los precios domésticos en los mismos términos es el inverso de los términos de intercambio. Puesto que suponemos inexistencia de bienes no comerciables, la relación mencionada es, en este caso, igual al tipo de cambio real. 


\subsection{La oferta agregada}

El análisis comienza suponiendo una ecuación de precios de bienes finales que depende directamente de los salarios nominales y del precio de los bienes externos, pues existen insumos importados

$$
p_{t}=f\left(e_{t}, w_{t}\right)
$$

Donde $p$ es el nivel de precios doméstico, $w$ el nivel de los salarios nominales, $e$ es el tipo de cambio nominal multiplicado por el nivel de precios externos, el cual se supone constante e igual a 1 .

El nivel de precios es homogéneo de grado uno en el precio de los insumos (salarios y tipo de cambio), y depende positivamente de cada uno de ellos.

A su vez, y siguiendo a Layard, Nickell y Jackman (1992, capítulo 8), existe una ecuación de salarios de la siguiente forma (ver también Blanchflower y Oswald, 1994).

$$
w_{t}=h\left(p_{t}, e_{t}, y_{t}\right)
$$

Donde $y$ es el producto de la economía.

El salario nominal depende positivamente de todos los elementos de la función $h$ y es, además, homogéneo de grado uno en precios y tipo de cambio.

Sustituyendo (2) en (1) se obtiene

$$
p_{t}=f\left(h\left(p_{t}, e_{t} y_{t}\right), e_{t}\right)=g\left(p_{t}, e_{t}, y_{t}\right)
$$

Por las propiedades de homogeneidad de grado uno de (1) y (2), el nivel de precios también es homogéneo de grado uno en sí mismo y en el tipo de cambio.

De aquí que

$$
1=g\left(E_{t}, y_{t}\right)
$$

Donde $E$ es el tipo de cambio real $e / p$.

Por el teorema de la función implícita es posible deducir que el producto $y$ es una función inversa del tipo de cambio real $E$, la cual linearizamos por conveniencia: 


$$
y_{t}=A-B E_{t}
$$

(5) es la oferta agregada que depende negativamente del tipo de cambio real.

La lógica del signo de la oferta agregada es la siguiente: si el tipo de cambio real aumenta, el precio relativo de los insumos importados también se incrementa y eso reduce la oferta de bienes.

A diferencia de una economía cerrada, una economía emergente que utiliza bienes importados en su producción tiene una oferta de largo plazo que no es vertical en el largo plazo. Esto se debe a que la oferta de insumos importados es, para un determinado tipo de cambio real, perfectamente elástica. En términos de la economía emergente no puede haber pleno empleo de los insumos importados. Este resultado, el cual también ha sido esbozado por Layard, Nickell y Jackman (1992, capítulo 8), es crucial en la obtención de los resultados posteriores.

\subsection{La demanda agregada}

El bloque de demanda agregada supone, primero, una función del tipo IS de la siguiente forma:

$$
y_{t}=b_{0}+b_{1} y_{t}-b_{2}\left(R_{t}-\pi_{t}\right)-b_{3} y_{t}+b_{4} E_{t}+b_{5} y_{t}^{*}
$$

$R$ es la tasa nominal de interés, $\pi$ la tasa de inflación y $y^{*}$ el producto externo. El parámetro $b_{1}$ es menor a uno y refleja la propensión marginal a gastar. El término $b_{5} y^{*}+b_{4} E-b_{3} y$ es la balanza comercial de la economía.

El parámetro $b_{0}$ refleja elementos exógenos de la demanda, como el gasto público, el cual influye positivamente sobre $b_{0}$, o los impuestos tipo lump sum, los cuales tienen un efecto negativo sobre el parámetro. Este parámetro $b_{0}$ también podría reflejar lo que en el modelo keynesiano básico eran el consumo autónomo y la inversión autónoma.

La demanda agregada depende negativamente de la tasa real de interés $(R-\pi)$ y positivamente del tipo de cambio real y del producto externo.

Solucionando para el producto doméstico se obtiene que

$$
y_{t}=\frac{b_{0}}{1-b_{1}+b_{3}}-\frac{b_{2}}{1-b_{1}+b_{3}}\left(R_{t}-\pi_{t}\right)+\frac{b_{4}}{\left(1-b_{1}+b_{3}\right)} E_{t}+\frac{b_{5}}{1-b_{1}+b_{3}} y_{t}^{*}(7)
$$




$$
\frac{\partial y_{t}}{\partial E_{t}}=\frac{b_{4}}{1-b_{1}+b_{3}}
$$

La ecuación de la balanza comercial en equilibrio es

$$
\begin{gathered}
b_{5} y_{t}^{*}+b_{4} E_{t}-b_{3} y_{t}=0 \\
\frac{\partial y_{t}}{\partial E_{t}}=\frac{b_{4}}{b_{3}}
\end{gathered}
$$

Lo anterior implica que en el plano donde se grafican el tipo de cambio real en el eje vertical y el producto en el eje horizontal, la ecuación de la demanda agregada tiene mayor pendiente que la de la balanza comercial.

Por otra parte, en el mercado monetario el modelo supone una demanda estable por dinero, la cual depende positivamente del ingreso y negativamente de la tasa de interés nominal $R$.

$$
\frac{M_{t}}{P_{t}}=a_{1} y_{t}-a_{2} R_{t}
$$

Donde $M$ es la cantidad nominal de dinero.

\subsection{La paridad de tasas de interés}

El modelo supone perfecta movilidad de capitales, por lo cual hay paridad de tasas de interés.

La tasa doméstica de interés es

$$
R_{t}=R_{t}^{*}+\frac{1}{e_{t}} \frac{d e}{d t}
$$

Donde (1/e)de/dt es la tasa de devaluación del tipo de cambio nominal. $R^{*}$ es la tasa nominal de interés externa.

Esta condición se puede sustituir en la ecuación de la IS, obteniéndose así

$$
y_{t}=h_{0}+h_{4} E_{t}-h_{2} R_{t}^{*}-h_{2}\left(\frac{1}{e_{t}} \frac{d e}{d t}-\pi_{t}\right)+h_{5} y_{t}^{*}
$$

Donde 


$$
h_{x}=\frac{b_{x}}{1-b_{1}+b_{3}} \quad \mathrm{x}=0,2,4,5
$$

\subsection{Inestabilidad intrínseca en el modelo}

La ecuación de la IS y la oferta agregada contienen los elementos necesarios que resuelven simultáneamente para el producto $y$ y el tipo de cambio real $E$.

Si los precios domésticos son completamente flexibles, cualquier régimen cambiario provee los mismos resultados para las variables reales.

La igualdad entre la oferta agregada (5) y la ecuación IS (13) da por resultado la ecuación diferencial:

$$
\frac{1}{e_{t}} \frac{d e}{d t}-\pi_{t}=\frac{h_{0}}{h_{2}}-\frac{A}{h_{2}}-R_{t}^{*}+\frac{h_{5}}{h_{2}} y_{t}^{*}+\frac{\left(B+h_{4}\right)}{h_{2}} E_{t}
$$

(14) resuelve para el tipo de cambio real E. Es una ecuación inestable en el sentido tradicional de la palabra. La derivada del tipo de cambio con respecto al tiempo $((1 / e)(d e / d t)-\pi)$ depende positivamente del nivel del tipo de cambio real $E$.

En términos de los modelos de expectativas racionales, el hecho de observar cierta inestabilidad en un grupo de ecuaciones e inestabilidad total si hay una sola ecuación es una característica deseable (ver Sargent y Wallace, 1973; Dorbusch, 1976; Blanchard y Fischer, 1989; Woodford, 2001) por dos razones fundamentales: primero porque permite llevar a cabo ejercicios donde las expectativas intervienen en el modelo; segundo porque si la ecuación fuera estable en el sentido tradicional, cualquier nivel inicial del tipo de cambio real llevaría al sistema al equilibrio, por lo cual la condición inicial del sistema estaría indeterminada y estrictamente habría infinitas soluciones. ${ }^{4}$

Como el modelo es intrínsecamente inestable, las soluciones para cambios inesperados en las variables exógenas se resuelven en el estado estacionario en cambios instantáneos de las variables endógenas. De otra manera el modelo nunca podría alcanzar equilibrio (ver Sargent y Wallace, 1973; Blanchard y Fischer, 1989, capítulo 5).

De lo anterior resulta que si los cambios de las variables exógenas son inesperados, el modelo dinámico de economía abierta y pequeña se convierte en un modelo estático de fácil solución gráfica.

4 Dornbusch (1976) encuentra también una ecuación inestable para el tipo de cambio nominal. 
1.5 Solución del modelo ante cambios inesperados de las variables fiscales y del producto externo

Si una variable exógena del modelo cambia inesperadamente y para siempre, entonces la única solución viable es un cambio de una vez por todas en el tipo de cambio real de la ecuación diferencial (14). De esta forma, los cambios continuos del tipo de cambio nominal son iguales a la inflación y los movimientos del tipo de cambio real para equilibrar oferta y demanda agregadas tienen lugar en forma instantánea.

$$
\frac{1}{e} \frac{d e}{d t}=\pi_{t}
$$

Lo anterior implica que, para cambios de la naturaleza mencionada (que son los típicos cambios que se investigan en un modelo estático), y dada la ecuación (15), es posible replantear las ecuaciones de oferta y demanda agregadas de la siguiente forma:

$$
\begin{gathered}
y_{t}=A-B E \\
y=h_{0}-h_{2} R^{*}+h_{4} E+h_{5} y^{*}
\end{gathered}
$$

Donde (16) es la oferta agregada que depende negativamente del tipo de cambio real y (17) la demanda dependiendo positivamente de la misma variable.

El sistema mencionado se puede resolver de una manera muy simple en forma gráfica (ver Gráfica 1).

$O A$ es la oferta agregada, $D A$ es la demanda agregada y $B C$ la balanza comercial de equilibrio, la cual tiene menor pendiente que la demanda agregada, tal como se vio anteriormente. En la Gráfica 1, el punto de equilibrio entre oferta y demanda agregadas también representa un punto de equilibrio de la balanza comercial. Por arriba de la recta de esta función hay superávit pues el tipo de cambio real es más alto y el producto más bajo, y por debajo de la recta hay déficit.

Los cambios en las variables exógenas desplazan las rectas. Así por ejemplo, una política fiscal expansiva, la cual ocurre por un incremento en el gasto público o por una reducción de impuestos, desplaza la demanda agregada $D A$ hacia la derecha, propiciando un menor tipo de cambio real y un mayor producto. Como la función de la balanza comercial $B C$ no se mueve, la política fiscal expansiva produce un déficit de la balanza comercial. 
Los efectos de la politica fiscal y el producto externo en una economía emergente abierta al exterior

Gráfica 1

Oferta y demanda agregadas y balanza comercial

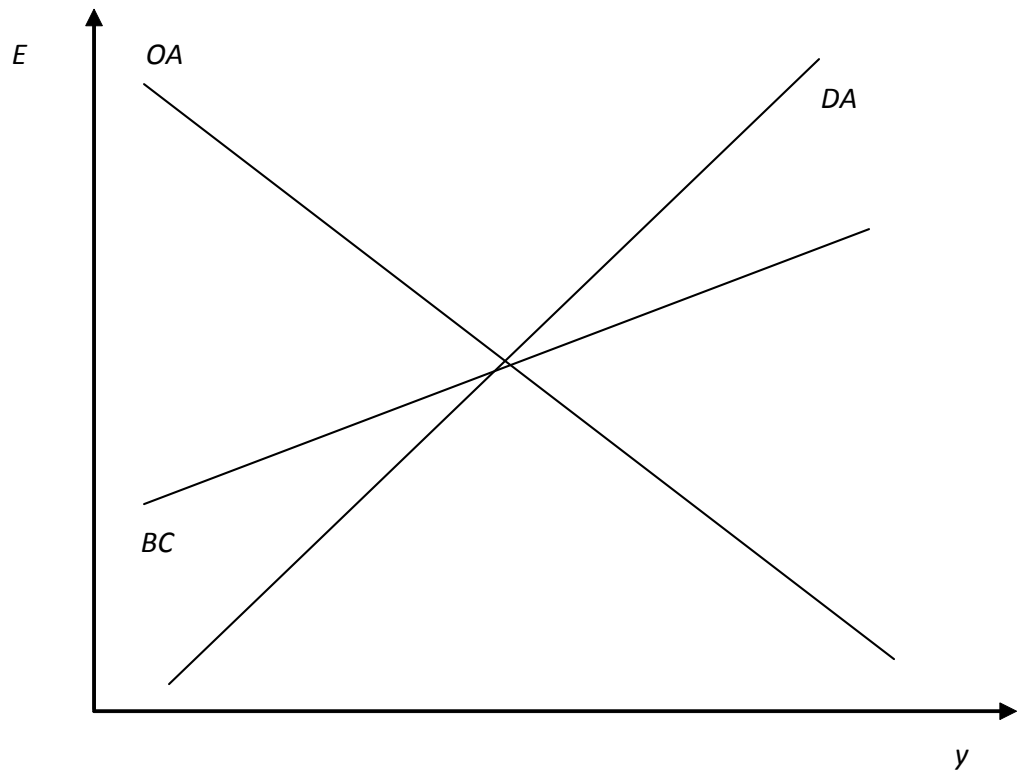

Fuente: elaboración propia.

Por su parte, un incremento del producto externo también desplaza la función $D A$ hacia la derecha, pero en esta ocasión la curva $B C$ se desplaza igualmente hacia abajo. Esto puede observarse al tomar la diferencial $d E / d y^{*}$ en la ecuación (9); el resultado es:

$$
\frac{d E}{d Y_{b c}^{*}}=-\frac{b_{5}}{b_{4}}
$$

Es posible demostrar que cuando $y^{*}$ cambia el desplazamiento vertical de la función $B C$ y el de la función $D A$ son iguales. Esto se observa al tomar la derivada $d E / d y^{*}$ en la ecuación de demanda (13). El resultado es:

$$
\frac{d E}{d Y_{i s}^{*}}=\frac{h_{5}}{h_{4}}=-\frac{b_{5}}{b_{4}}
$$

El hecho de que las dos derivadas en (18) y (19) sean iguales implica que el desplazamiento vertical de la $D A$ es igual al de la $B C$. Si eso sucede, y si en el punto 
original de equilibrio entre la oferta y la demanda agregadas también hay equilibrio en la balanza comercial, entonces en el nuevo punto de equilibrio entre oferta y demanda habrá superávit comercial, lo que se observa en la Gráfica 2:

\section{Gráfica 2}

Incremento del producto externo y su efecto sobre el producto, el tipo de cambio real y la balanza comercial en el corto plazo

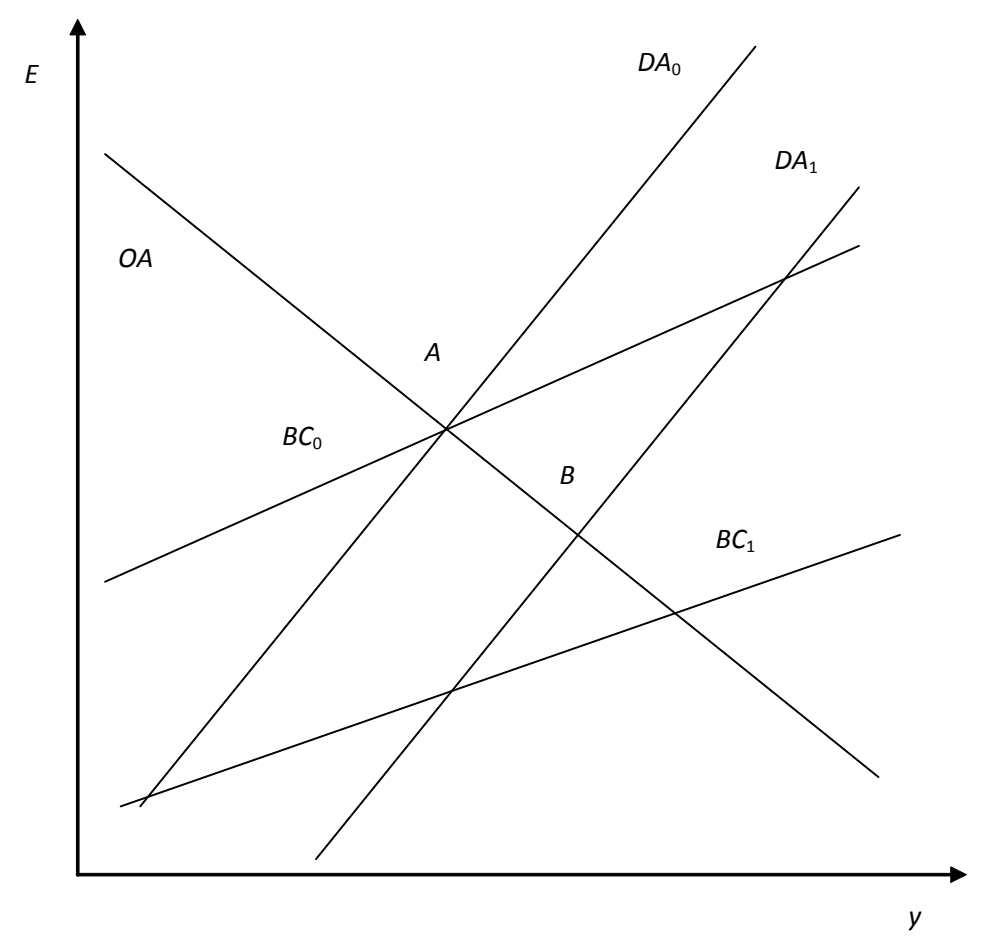

Fuente: elaboración propia.

El equilibrio inicial está en $A$, en la intersección de la oferta agregada $O A$ con la demanda $D A_{0}$. El incremento en el producto externo desplaza la $D A$ y la $B C$ hacia abajo, siendo idéntico el desplazamiento vertical de ambas funciones. El nuevo equilibrio entre oferta y demanda termina en $B$, por arriba de la nueva $B C_{1}$, por lo cual hay un superávit comercial.

En el corto plazo la política fiscal expansiva y el aumento del producto externo tienen igual efecto sobre el producto y el tipo de cambio real, aunque opuesto sobre la balanza comercial. 
1.6 El papel de la política monetaria y el régimen cambiario en el modelo estático

La política monetaria no tiene ningún efecto en las variables reales de este modelo básico si el nivel de precios es perfectamente flexible. Si las autoridades monetarias manejan la tasa de crecimiento del tipo de cambio nominal (1/e)(de/dt), la inflación será igual a dicha tasa de crecimiento y los cambios abruptos en el tipo de cambio real tendrán lugar a través de movimientos de una vez por todas en el nivel general de precios.

Cuando el gobierno aumenta el gasto público (el parámetro $h_{0}$ ), el producto aumenta y el tipo de cambio real disminuye, lo cual ocurre a través de un incremento de una vez por todas en el nivel general de precios.

Sin embargo, este modelo admite la posibilidad de contar con una política donde el tipo de cambio sea flexible. Una posibilidad de plantear esta política sería asumiendo la existencia de una regla de Taylor para tasas de interés (ver Taylor, 1993; Romer, 2000; Woodford, 2001). Siguiendo a Romer (2000), las autoridades monetarias fijan la tasa nominal de interés a través del siguiente mecanismo

$$
R_{t}=Z+(1+\Omega) \pi_{t}
$$

Donde $Z$ es un parámetro discrecional de política monetaria que si aumenta eleva las tasas de interés nominales ex-ante. La política de tasas de interés se sobreajusta a la inflación ( $\Omega$ es mayor a cero). En el caso más simple, que es el que en general analiza Romer (2000), esta regla no depende del producto. ${ }^{5}$

Al igualar (20) con la paridad de tasas de interés e imponer (15), porque sólo hay cambios inesperados de una vez por todas en las variables exógenas, se obtiene que

$$
\pi=\frac{R^{*}-Z}{\Omega}
$$

La inflación depende positivamente de la tasa de interés externa y negativamente del parámetro de política monetaria $Z$ y del producto.

5 Sería posible asumir la regla $R_{t}=Z+(1+\Omega) \pi_{t}+\gamma\left(y_{t}^{d}-y_{t}^{s}\right)$, donde $y_{t}^{d}$ es la demanda agregada y $y_{t}^{s}$ es la oferta agregada. $y_{t}^{d}-y_{t}^{s}$ es el exceso de demanda de la economía. Bajo el supuesto de equilibrio $y_{t}^{s}=y_{t}^{d}$, la regla converge al resultado de la ecuación (20). 
Si en el modelo de oferta y demanda agregada se lleva a cabo una política fiscal expansiva entonces, con un régimen de tipo de cambio flexible y una política monetaria que utiliza una regla de Taylor, el producto aumenta, el tipo de cambio disminuye, se genera un déficit comercial y la inflación permanece constante.

En regímenes de tipo de cambio flexible, un punto de gran importancia a considerar es cómo se ajusta el tipo de cambio real si tanto el tipo de cambio nominal como el nivel de precios son totalmente flexibles. La respuesta se obtiene analizando el mercado de dinero. La ecuación diferencial (14) resuelve para el tipo de cambio real. La oferta agregada (5) resuelve entonces para el producto. Dada esta solución, (21) resuelve para la inflación. En el contexto de una política monetaria que utiliza tasas de interés, el banco central surte toda la demanda de dinero deseada a través de la oferta nominal de dinero a precios predeterminados. Así que, si el gasto público o el producto externo aumentan, el producto doméstico aumenta y la tasa nominal de interés permanece constante. La ecuación (11) del mercado de dinero indica que, a los precios dados en ese instante el banco central incrementa la oferta nominal $M$ para que el mercado de dinero siga estando en equilibrio. De modo que, en este caso el nivel de precios queda predeterminado y sigue creciendo a la misma tasa de inflación. Por su parte, el tipo de cambio real se ajusta a través de una caída de una vez por todas en el tipo de cambio nominal.

La intuición del motivo por el cual una política fiscal expansiva o un incremento del producto externo reducen el tipo de cambio nominal es idéntica a la que surge en el modelo Mundell-Fleming (ver Mundell, 1968). Con perfecta movilidad de capitales un choque positivo a la curva IS presiona las tasas de interés domésticas hacia arriba. Sin embargo, con tipo de cambio flexible y altísima movilidad de capitales el incremento en las tasas domésticas no llega a concretarse. La solución es una caída en el tipo de cambio nominal por una entrada intencional de capitales, que surge de la presión hacia arriba de las tasas de interés domésticas.

\section{La restricción de la cuenta corriente, la equivalencia ricardiana y el equilibrio de largo plazo}

El modelo anterior puede criticarse porque, aunque la paridad de tasas de interés garantiza equilibrio en la balanza de pagos, no hay un mecanismo de corrección para el desajuste de la balanza comercial y la cuenta corriente. Este mismo problema ocurre

prácticamente en todos los modelos keynesianos del sector externo, como sería el 
caso del modelo Mundell-Fleming (Mundell, 1968), el modelo de Blanchard y Fischer (1990, p. 537-540) o el modelo de Romer (2000). ${ }^{6}$

En el modelo descrito en la sección anterior, si estando en equilibrio el gasto público aumenta, el nuevo equilibrio de largo plazo implica un déficit permanente en balanza comercial. Si este argumento se utiliza con la cuenta corriente el resultado es que, en una circunstancia como la descrita habría una situación explosiva para dicha cuenta pues, para financiarla habría que endeudarse más y más propiciando mayor pago de intereses, mayor déficit en cuenta corriente y así sucesivamente. Si hubiera un sistema de tipo de cambio flexible, la cuenta de capital sería un espejo de la cuenta corriente.

Una pregunta relevante es si el modelo de estructura keynesiana, que presentamos en este artículo, puede transformarse en un modelo de otro tipo en el cual los movimientos de ciertas variables exógenas garanticen el equilibrio de la cuenta corriente, o de alguna variación de dicho concepto, en el largo plazo.

Para contestar lo anterior, haremos un supuesto adicional sobre la demanda agregada inicial que implica que ésta dependa positivamente de los activos financieros externos totales que tiene la economía. Dichos activos son los bonos externos en poder de los sectores público y privado, mismos que pueden ser negativos, en cuyo caso el país estaría endeudado y las reservas internacionales en poder del banco central. Los bonos domésticos en poder de extranjeros entran como pasivos de los residentes domésticos y por tanto ejercen un efecto negativo sobre la demanda agregada.

La razón para incluir estos activos es, que si los agentes económicos privados tienen una visión de largo plazo entonces su gasto dependerá de dichos activos porque percibirán que la economía tiene que ajustarse cuando está fuertemente endeudada, o podrá haber mayor gasto en general si los activos son muy elevados. Asimismo, el sector público consolidado con el banco central tenderá a reducir el gasto o subir los impuestos si observa que los activos externos son muy bajos.

En modelos de optimización dinámica con horizontes infinitos de tiempo, los agentes económicos ajustan su consumo de modo que la cuenta corriente de largo plazo esté en equilibrio. Cuando hay perfecta movilidad de capitales y el consumo tiene una trayectoria plana, ${ }^{7}$ al igual que las variables exógenas, la cuenta corriente

Igualmente para el modelo de Dornbusch (1976) o el de Layard, Nickell y Jackman (1992).

Para que el consumo tenga una trayectoria plana diversos autores suponen que la tasa de interés externa es igual a la tasa de preferencias intertemporales (ver por ejemplo Obstfeld, 1985; Drazen y Helpman, 1987; Calvo, 1986). Otros autores hacen endógena la tasa de preferencias intertemporales (Blanchard, 1985). 
siempre está en equilibrio y ocurre la equivalencia ricardiana (ver Barro, 1974), una situación donde los impuestos actuales no tienen ningún efecto sobre el consumo privado y donde movimientos del consumo público generan un efecto de desplazamiento (crowding out) perfecto sobre el consumo privado (ver por ejemplo Obstfeld, 1985; Calvo, 1986; Drazen y Helpman, 1987).

La experiencia de muchos países es que, aunque el gasto público, los impuestos y el consumo mantengan niveles relativamente constantes (al menos como proporción del producto), la cuenta corriente muestra fuertes desequilibrios, por lo que los modelos de optimización dinámica con horizontes infinitos de tiempo no parecen ser una buena descripción de la realidad. Autores como Blanchard (1985) y Helpman y Razin (1987) trabajan con modelos de horizontes finitos en los cuales la equivalencia ricardiana no ocurre.

En el caso que nos ocupa, introducir los activos externos como determinante del gasto en la demanda agregada es una forma de generar algún tipo de ajuste ricardiano de largo plazo. En un momento dado, un aumento en el gasto público puede producir una expansión y un déficit en cuenta corriente porque los agentes económicos de la actualidad tienen vidas finitas y el nuevo endeudamiento no tendrá efecto en su horizonte de tiempo. Sin embargo, conforme va pasando el tiempo los nuevos agentes que van apareciendo se dan cuenta de que cargan con una deuda importante y comienzan a reducir su gasto para afrontar el necesario ajuste del futuro. Esta idea es muy similar a la de Helpman y Razin (1987).

\subsection{Solución del modelo cuando la}

demanda agregada depende de los activos financieros externos

La demanda agregada, en forma ya reducida (como en la ecuación (13)), quedaría como sigue:

$$
y_{t}=h_{0}+h_{4} E_{t}-h_{2} R_{t}^{*}-h_{2}\left(\frac{1}{e_{t}} \frac{d e}{d t}-\pi_{t}\right)+h_{5} y_{t}^{*}+h_{6}\left(E f-b_{n e t}\right)
$$

Donde $f$ son los activos financieros externos valuados en moneda o bienes externos, por lo cual para pasarlos a bienes domésticos hay que multiplicarlos por el tipo de cambio real. El término $b_{n e t}$ representa los bonos en poder de extranjeros que el gobierno doméstico u otras agencias privadas nacionales emiten. El parámetro $h_{6}$ 
muestra el efecto neto $\left(h_{6}=b_{6} / b_{2}\right)$ de los activos externos netos totales sobre la demanda agregada, el cual es positivo.

La demanda agregada (22) se iguala ahora a la oferta (5) para obtener la ecuación dinámica del tipo de cambio real:

$$
\frac{1}{e_{t}} \frac{d e}{d t}-\pi_{t}=\frac{h_{0}}{h_{2}}-\frac{A}{h_{2}}-R_{t}^{*}+\frac{h_{5}}{h_{2}} y_{t}^{*}+\frac{\left(B+h_{4}\right)}{h_{2}} E_{t}+\frac{h_{6}}{h_{2}}\left(E f-b_{n e t}\right)
$$

El modelo se cierra a través de la ecuación de la cuenta corriente, la cual es simplemente la suma de la balanza comercial más la balanza de servicios factoriales, en este caso el pago de intereses por los activos financieros del exterior.

En este punto surge un problema porque distintos activos externos pagan distintas tasas de interés. Por ejemplo, las reservas internacionales y los bonos externos y/o los créditos que diversas agencias públicas y privadas adquieren del exterior pagan la tasa externa de interés exclusivamente (el saldo de crédito entraría como signo negativo para $f$ ). ${ }^{8}$ Sin embargo, los bonos que el gobierno u otras agencias privadas emiten a los extranjeros pagan tasas nacionales que están valuadas en moneda o bienes domésticos.

De esta forma, la cuenta corriente de la balanza de pagos se puede expresar como

$$
b_{5} y_{t}^{*}+b_{4} E_{t}-b_{3} y_{t}+R_{t}^{*} E_{t} f_{t}-\left(R_{t}^{*}+\frac{1}{E} \frac{d e}{d t}-\pi_{t}\right) b_{n e t}=E_{t} \frac{d f_{t}}{d t}-\frac{d b_{n e t}}{d t}
$$

Donde $f$ son las reservas internacionales más los bonos externos que compran los nacionales en el exterior, a estos se les resta el saldo de crédito que diversas agencias domésticas adquieren directamente en el exterior. Estos activos netos están valuados en moneda extranjera; $b_{n e t}$ son los bonos domésticos en términos reales que emite el gobierno a extranjeros.

Una solución relativamente tratable del problema consiste en asumir que los bonos domésticos que el gobierno emite a extranjeros son cero. En este caso al sustituir (5) en (24), esta última se convierte en

$$
b_{5} y_{t}^{*}+\left(b_{4}+b_{3} B\right) E_{t}-b_{3} A+R_{t}^{*} E_{t} f_{t}=E_{t} \frac{d f_{t}}{d t}
$$

\footnotetext{
8 Por ejemplo préstamos del Fondo Monetario Internacional o el Banco Mundial.
} 
Sustituyendo el valor de las $h$ 's en la demanda agregada por sus valores originales en términos de las $b$ 's (ver ecuaciones (7) y (13)) y valuando (23) y (25) en el estado estacionario, se obtiene una aproximación lineal del sistema que se describe como

$$
\frac{1}{e} \frac{d e}{d t}-\pi_{t}=\left(\frac{b_{4}+\left(1-b_{1}+b_{3}\right) B+b_{6} f_{0}}{b_{2}}\right) E+\frac{b_{6}}{b_{2}} E_{0} f+\frac{b_{0}}{b_{2}}-\frac{A\left(1-b_{1}+b_{3}\right)}{b_{2}}-\left(R_{t}^{*}+\sigma\right)+\frac{b_{5}}{b_{2}} y_{t}^{*}
$$

$$
E_{t} \frac{d f_{t}}{d t}=\left(b_{4}+b_{3} B+R_{t}^{*} f_{0}\right) E_{t}+R_{t}^{*} E_{0} f+b_{5} y_{t}^{*}-b_{3} A
$$

Donde $f_{0}$ y $E_{0}$ son los valores de $f$ y $E$ en el estado estacionario. Un supuesto será que los términos $b_{4}+B\left(1-b_{1}+b_{3}\right)+b_{6} f_{0}$ y $b_{4}+b_{3} B+R^{*} f_{0}$ son mayores a cero. Esto definitivamente es cierto si $f_{0}$ es mayor o igual a cero y no se cumplirá sólo si $f_{0}$ es muy negativo.

El supuesto de estos dos valores positivos es para preservar el efecto positivo del tipo de cambio real tanto sobre la demanda agregada como sobre la cuenta corriente. Si el término $b_{4}+B\left(1-b_{1}+b_{3}\right)+b_{6} f_{0}$ fuera negativo, entonces una deuda muy elevada ( $f_{0}$ fuertemente negativa) daría lugar a que el aumento del tipo de cambio real tuviera un efecto riqueza muy negativo y redujera, en vez de incrementar, la demanda agregada. Si el término $b_{4}+b_{3} B+R^{*} f_{0}$ fuera negativo, el aumento del tipo de cambio real deterioraría de tal forma el valor actual de la deuda que tendría un efecto negativo sobre la cuenta corriente. Esto sería como una violación de la condición de Marshall-Lerner sobre la cuenta corriente.

El determinante del sistema (26) y (27) es ${ }^{9}$

$$
\operatorname{Det}=\left(\frac{b_{4}+B\left(1-b_{1}+b_{3}\right)+b_{6} f_{0}}{b_{2}}\right) R_{t}^{*} E_{0}-\frac{b_{6}}{b_{2}} E_{0}\left(b_{4}+b_{3} B+R_{t}^{*} f_{0}\right)
$$

Este determinante puede ser positivo o negativo. Si es positivo, el sistema es o globalmente inestable o globalmente estable. Ambos casos son difíciles de tratar en términos económicos, pues el tipo de cambio real es perfectamente flexible pero

9 Estamos asumiendo que $b_{4}+\left(1-b_{1}+b_{3}\right)+b_{6} f_{0}>b_{4}+b_{3} B+R^{*} f_{0}>0$. Esto definitivamente sucede cuando $f_{0}>0$ si además $b_{6}>R^{*}$ pero podría no ocurrir en un país muy endeudado donde $f_{0}$ es muy negativo. Para países con deuda relativamente baja el supuesto sigue siendo razonable. 
los activos externos están predeterminados y sólo varían a lo largo del tiempo. Si el sistema fuera globalmente inestable, carecería de solución y si fuera globalmente estable tendría infinitas soluciones.

Sin embargo, si el determinante es negativo el equilibrio es de punto silla e implica que

$$
\frac{b_{6} E_{0}}{b_{4}+B\left(1-b_{1}+b_{3}\right)+b_{6} f_{0}}>\frac{R_{t}^{*} E_{0}}{b_{4}+b_{3} B+R_{t}^{*} f_{0}}
$$

Pero esta condición se resume simplemente en que el valor absoluto de la pendiente $d E / d f$ en el estado estacionario de la ecuación (26), que es igual al término del lado izquierdo en la ecuación (29), es mayor que el valor absoluto de la pendiente $d E / d f$ en el estado estacionario para la ecuación (27), que es igual al término del lado derecho en la ecuación (29). Ambas pendientes son negativas.

Para que esta condición tenga lugar lo importante es, que el efecto de los activos externos sobre la demanda agregada $\left(b_{6}\right)$ sea suficientemente elevado. De hecho, debe ser suficientemente mayor que la tasa de interés externa $R^{*}$, pues si fueran iguales la condición (29) no se cumpliría pues el denominador del término del lado derecho de tal ecuación es menor que el denominador del término del lado izquierdo de la misma.

Si el efecto de los activos externos sobre la demanda agregada es suficientemente elevado, habrá una trayectoria de punto silla en el sistema, la cual llevará al tipo de cambio real y a los activos externos hacia valores estables donde la cuenta corriente estará finalmente en equilibrio.

La Gráfica 3 muestra el equilibrio de punto silla entre el tipo de cambio real $E$ y los activos externos $f$ con la trayectoria de punto silla

La recta eq con mayor pendiente en valor absoluto representa a la ecuación (26), o el equilibrio entre la oferta y la demanda agregada del sistema. La recta $c c$, con menor pendiente en valor absoluto, representa el equilibrio de la cuenta corriente de la ecuación (27).

Diversos ejercicios muestran cómo se ajusta el modelo en corto y largo plazo. El primero es una política fiscal expansiva, cuyos efectos pueden observarse en la Gráfica 4.

El equilibrio inicial está en $A$, en el cruce de la ecuación $e q_{0}$ de equilibrio de oferta y demanda con la ecuación $c c$ de equilibrio en la cuenta corriente. Si el gasto público aumenta (el parámetro $b_{0}$ en (26) sube), la ecuación $e q_{0}$ se desplaza hacia 


\section{Gráfica 3}

Equilibrio de punto silla entre el

equilibrio del mercado de bienes y la cuenta corriente en la balanza de pagos

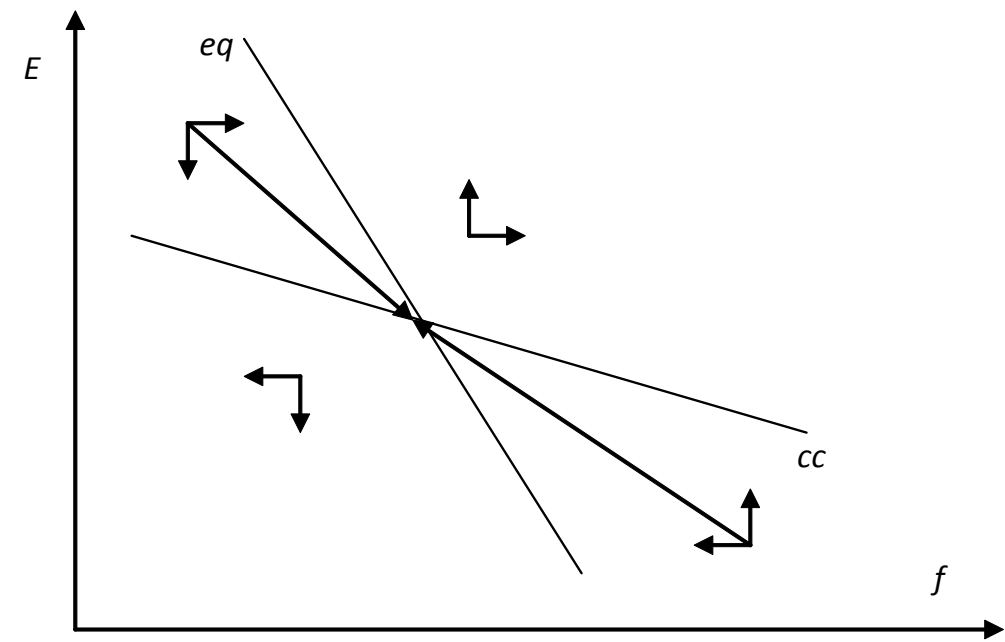

Fuente: elaboración propia.

\section{Gráfica 4}

Efectos de corto y largo plazo de una política fiscal expansiva sobre el tipo de cambio real y los activos externos netos

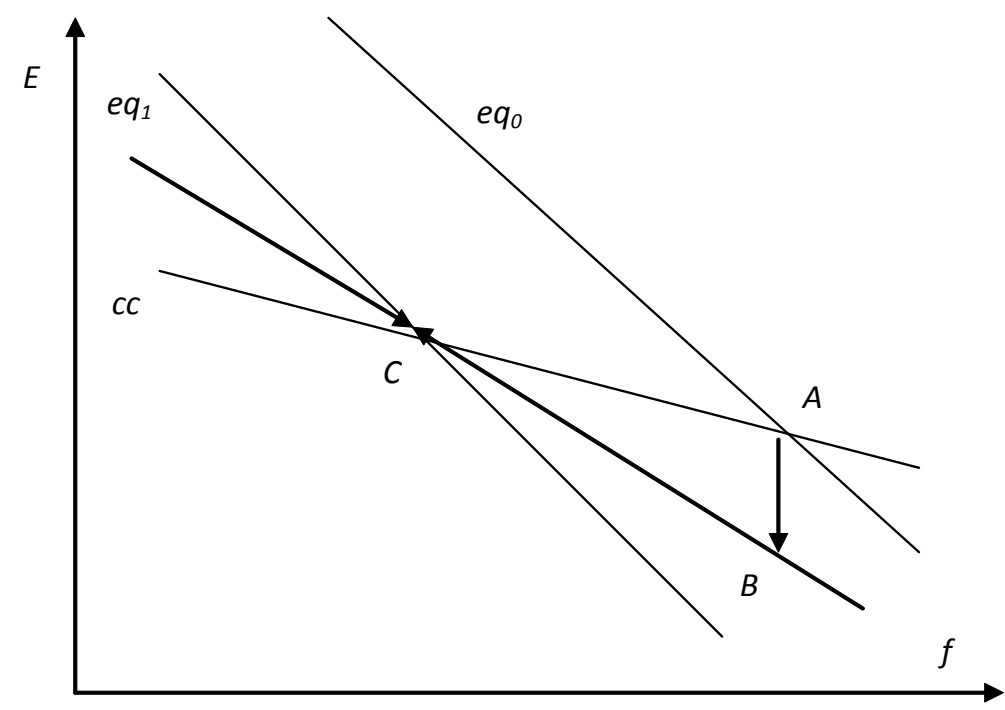

Fuente: elaboración propia. 
abajo pues, de acuerdo a la ecuación (26), un incremento del gasto requiere una reducción del tipo de cambio real para que el sistema siga en el estado estacionario.

Inicialmente, dado que $f$ es una variable predeterminada y $E$ no lo es, hay una fuerte apreciación del tipo de cambio real, el cual baja de $A$ a $B$ desde el equilibrio inicial hasta la nueva trayectoria de punto silla. En $B$, sin embargo, el tipo de cambio real comienza a depreciarse en el tiempo. El sistema termina en el punto $C$, con un tipo de cambio real más depreciado y menores activos externos.

Lo anterior implica que en el corto plazo hay una expansión del producto (ver oferta agregada (5)) y un déficit en cuenta corriente. El desajuste externo propicia una depreciación lenta del tipo de cambio real, la cual comienza a reducir el producto. La cuenta corriente vuelve al equilibrio con un tipo de cambio real, también de equilibrio, más elevado. Esto último, por la ecuación de oferta agregada, implica que el producto final está por debajo del inicial.

La recesión en la que finalmente entra la economía ocurre porque el déficit en cuenta corriente produce una caída en los activos externos, lo que propicia que la demanda comience a ajustarse a la baja generando una reducción de la actividad económica.

El otro ejercicio a considerar es el aumento de la actividad económica externa (un incremento en $y^{*}$ ). Dicho incremento produce cambios en las dos ecuaciones. En la ecuación de equilibrio entre oferta y demanda agregada (26), el efecto inicial de $y^{*}$ sobre el tipo de cambio real $E$ es:

$$
\frac{d E}{d y_{e q}^{*}}=\frac{-b_{5}}{b_{4}+B\left(1-b_{1}+b_{3}\right)+b_{6} f_{0}}
$$

Asimismo, el efecto inicial de $y^{*}$ sobre el tipo de cambio real en la ecuación de equilibrio de la cuenta corriente es:

$$
\frac{d E}{d y_{c c}^{*}}=\frac{-b_{5}}{b_{4}+B b_{3}+R^{*} f_{0}}
$$

En equilibrio, la ecuación eq en el plano $(f, E)$ se desplaza hacia abajo menos que la $c c$ pues para que exista una trayectoria de punto silla el denominador de (30) tiene que ser mayor que el de (31).

En términos gráficos, lo anterior implica que un aumento del producto externo aprecia el tipo de cambio real y genera un superávit en cuenta corriente, el cual final- 
mente eleva los activos externos y propicia un impulso de largo plazo en el producto doméstico (ver Gráfica 5).

\section{Gráfica 5}

Efectos de corto y largo plazo de un incremento del producto externo sobre los activos externos netos y el tipo de cambio real

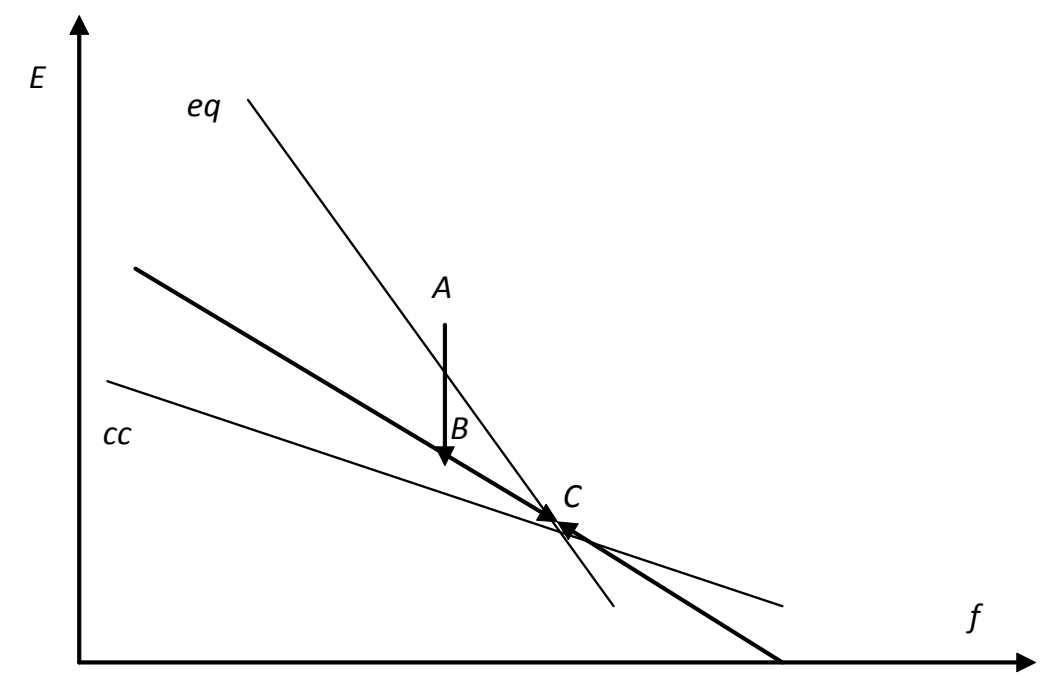

Fuente: elaboración propia.

Originalmente el equilibrio está en el punto $A$, en el cruce de dos ecuaciones no mostradas en el dibujo. El incremento de $y^{*}$ desplaza las dos ecuaciones $e q$ y $c c$ hacia abajo, pero la $c c$ más que la eq. Como los activos externos están predeterminados, el tipo de cambio real se ajusta de una sola vez de $A$ a $B$, donde está la nueva trayectoria de punto silla y de ahí en el tiempo la moneda continúa apreciándose hasta el nuevo equilibrio $C$, donde termina el superávit de cuenta corriente.

El producto se incrementa en el instante inicial en que se modifica el tipo de cambio real, pero continúa aumentando mientras la moneda sigue apreciándose.

La política monetaria continúa siendo neutral. En esta versión del modelo con una trayectoria de punto silla la solución para la inflación, que surge de igualar la ecuación de paridad de tasas de interés (12) con la regla de Taylor (18), queda como: 


$$
\pi_{t}=\frac{R^{*}+\left(\frac{1}{e} \frac{d e}{d t}-\pi_{t}\right)-Z}{\Omega}
$$

Así que, lo que sucede en el modelo de las ecuaciones (26) y (27) afecta a la inflación en la ecuación (32), pero la política monetaria representada por los parámetros $Z$ y $\Omega$ no afecta el modelo de las mencionadas ecuaciones. En el caso de una política fiscal expansiva, la inflación sube originalmente porque la moneda se está depreciando, pero este efecto sólo es temporal porque al final la depreciación es cero. En lo que respecta al producto externo, la inflación baja también temporalmente porque la moneda se está apreciando.

La Gráfica 6 muestra el comportamiento del producto, el tipo de cambio real y la inflación cuando hay una política fiscal expansiva y, una política monetaria a través de una regla de Taylor. El producto aumenta con rapidez y luego comienza a bajar hasta terminar en un nivel menor al original, lo contrario pasa con el tipo de cambio real. La inflación sube por la depreciación continua del tipo de cambio, la cual poco a poco se desacelera y propicia un retorno al nivel original.

\section{Gráfica 6}

Efectos de corto y largo plazo de una política fiscal expansiva

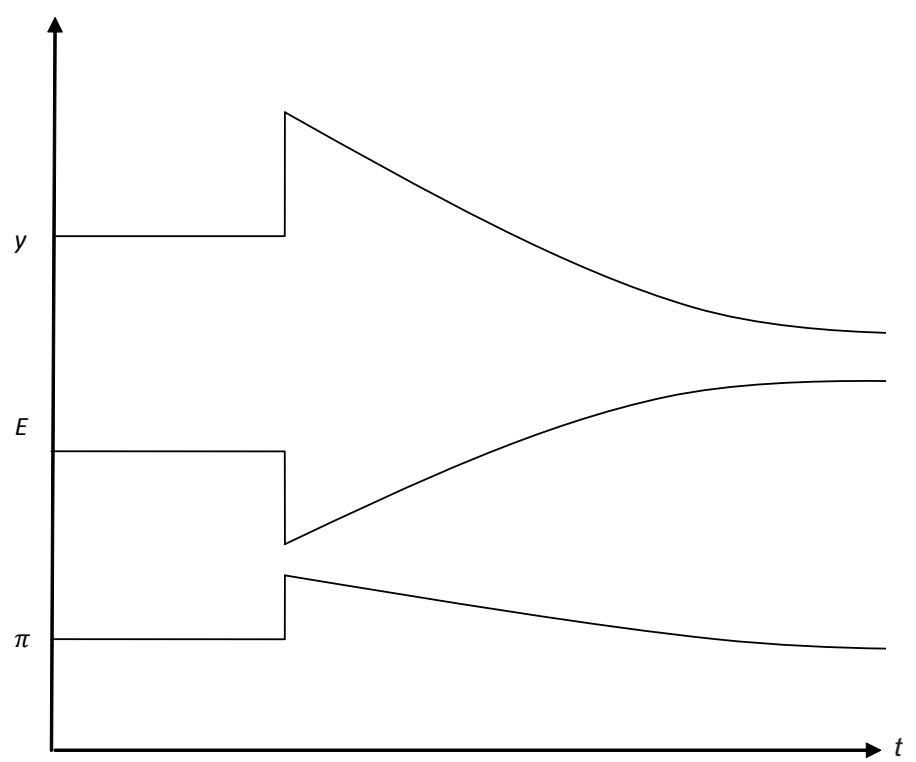

Fuente: elaboración propia. 
Por su parte, la Gráfica 7 muestra el efecto sobre el producto, el tipo de cambio real y la inflación de un incremento del producto externo. En este caso el impacto inicial sobre el producto y el tipo de cambio real es muy similar al de la política fiscal expansiva, pero después cambia radicalmente. El superávit de balanza comercial y cuenta corriente que genera el incremento en el producto externo impulsa todavía más la demanda, lo que incrementa todavía más el producto y reduce el tipo de cambio real. Como la moneda doméstica se está apreciando, la inflación cae temporalmente.

\section{Gráfica 7}

Efectos de corto y largo plazo de un incremento en el producto externo

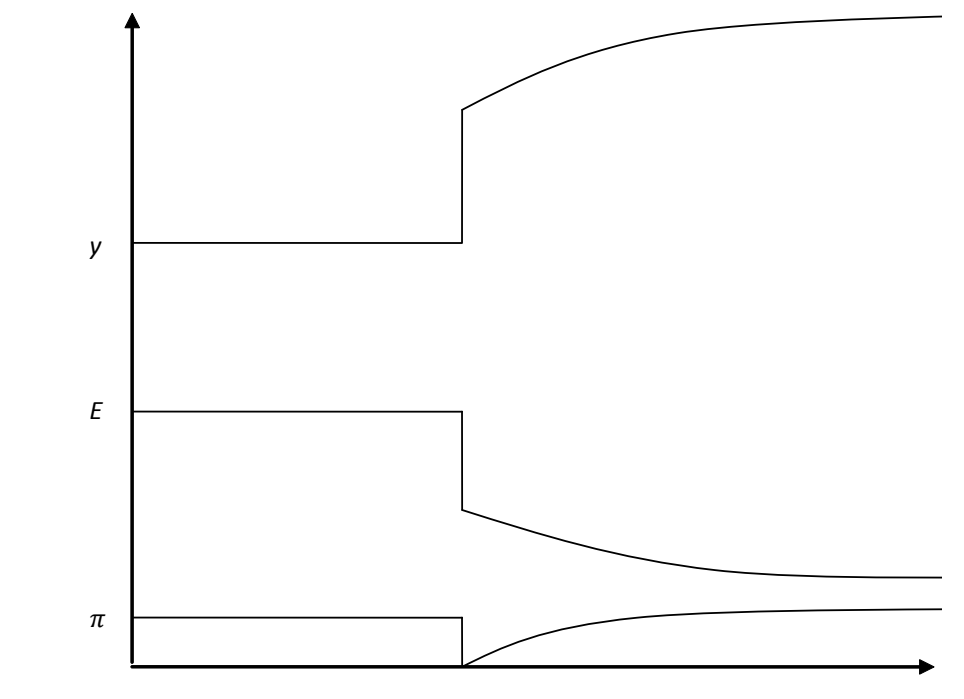

Fuente: elaboración propia.

\subsection{El uso de una política fiscal contracíclica en}

situaciones donde cae el producto externo

Si el producto externo cae, ¿qué efectos de corto y largo plazo tendría una política fiscal contracíclica? En el modelo de corto plazo una caída del producto externo propiciaría un efecto contrario al que se observa en la Gráfica 2: el producto doméstico caería, el tipo de cambio real aumentaría y la cuenta corriente entraría en un déficit. Una política fiscal contracíclica podría estabilizar el producto y el tipo de cambio 
real en el corto plazo al mover la demanda al punto inicial; sin embargo, eso provocaría un déficit de cuenta corriente todavía más elevado.

Los efectos de largo plazo de una política fiscal contracíclica serían altamente recesivos. La Gráfica 8 muestra estos efectos utilizando el modelo de las ecuaciones (26) y (27):

La gráfica muestra tres casos posibles:

\section{Gráfica 8}

Efectos de largo plazo de una política fiscal contracíclica ante una reducción del producto externo

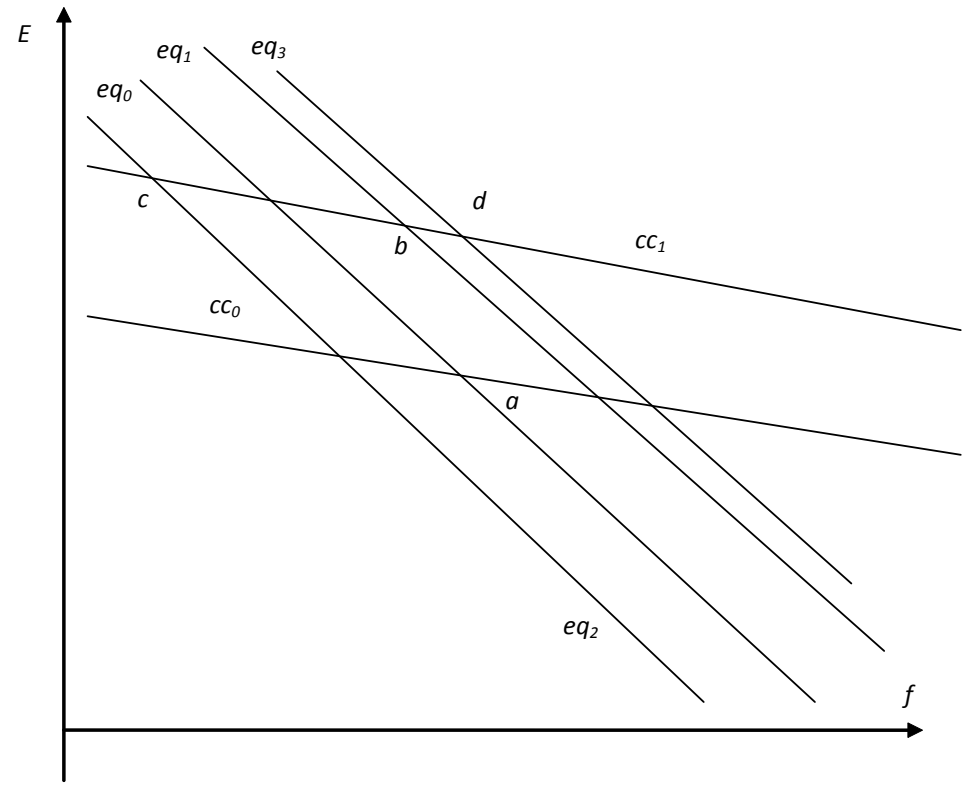

Fuente: elaboración propia.

El equilibrio inicial es en $a$, en la intersección de la función $e q_{0}$ con la $c c_{0}$. Si el producto externo cae y no hay intervención alguna de la autoridad fiscal, el nuevo equilibrio de largo plazo ocurre en $b$, en la intersección de la función $e q_{1}$ con la $c c_{1}$. Siguiendo las ecuaciones (30) y (31), la función $c c$ se desplaza más hacia arriba que la eq. En $b$ hay un tipo de cambio real más elevado y menores activos externos netos. 
Una política fiscal contracíclica consistiría en utilizar la política fiscal de manera que en el corto plazo la economía estuviera en $a$. Para eso la función eq se debería mover hacia abajo, de $e q_{0}$ a $e q_{2}$. La trayectoria de punto silla (no dibujada) pasaría exactamente por el punto $a$ y en el corto plazo no habría movimiento alguno ni del tipo de cambio real ni del producto. Sin embargo, esa política tendría un equilibrio de largo plazo en $c$, en donde habría un tipo de cambio real muy elevado y activos externos netos muy reducidos, lo que implicaría una fuerte contracción del producto de largo plazo.

Habría finalmente una última alternativa: una política fiscal procíclica. En este caso habría que acompañar a la reducción del producto externo con una política fiscal restrictiva, lo que movería la función $e q$ de $e q_{0}$ a $e q_{3}$, generando un desplazamiento vertical de dicha función igual al de la función $c c$. Esto mantendría constantes en todo momento los activos externos y subiría de inmediato el tipo de cambio real, causando una recesión desde el corto plazo. Sin embargo, esta alternativa, que dejaría el equilibrio en $d$ sería la que menor recesión de largo plazo causaría.

\section{Conclusiones}

Del análisis anterior hay una conclusión muy clara: en situaciones donde el producto externo cae, el uso de una política fiscal contracíclica puede atenuar o incluso anular los efectos recesivos del choque externo en el corto plazo, pero eso va a dar lugar a efectos de largo plazo muy recesivos sobre la economía emergente. En cambio, el uso de una política fiscal procíclica va a producir una recesión fuerte e inmediata de corto plazo pero va a ser la mejor opción para el producto en el largo plazo.

Lo anterior sucede porque, en corto plazo una caída en el producto externo propicia una reducción del producto doméstico, caída en el tipo de cambio real y déficit en cuenta corriente, mientras que una política fiscal expansiva propicia efectos contrarios para el producto doméstico y el tipo de cambio real pero también genera un déficit en cuenta corriente. El uso de una política fiscal contracíclica deteriora todavía más la cuenta corriente y eso es lo que da lugar a una fuerte recesión en el futuro. ${ }^{10}$

El tipo de ajuste de largo plazo aquí descrito ha sido analizado en la literatura. Sutherland (1995) propone que una deuda creciente puede reducir la demanda

10 El tipo de ajuste de largo plazo aquí descrito ha sido analizado en la literatura. Giavazzi y Pagano (1996) muestran diversos países en los cuales un ajuste fiscal genera una expansión y viceversa. 
agregada. Giavazzi y Pagano (1996) señalan que diversos países han experimentado efectos donde la política fiscal expansiva produce una recesión y viceversa.

Hay dos aspectos probablemente novedosos en el análisis de este trabajo: el primero es que la política fiscal y el producto externo tienen efectos sobre el producto doméstico aun en presencia de un tipo de cambio real que es perfectamente flexible. Esto sucede porque la oferta del producto doméstico tiene en todo momento pendiente positiva, lo cual, a su vez, ocurre por el uso de insumos intermedios importados en la producción doméstica. Estos insumos tienen una oferta perfectamente elástica para la economía emergente doméstica; no puede existir pleno empleo de insumos importados.

El hecho de que el tipo de cambio real sea perfectamente flexible para equilibrar siempre la oferta y la demanda propicia una política monetaria neutral, misma que no puede utilizarse para estabilizar el producto.

El segundo aspecto es que modelos de economía abierta estructurados a la manera keynesiana de los años ochenta y noventa, como los de Layard, Nickell y Jackman (1992) y Harrigan et al. (1993), podrían lograr que los activos externos alcanzaran un nivel de equilibrio, lo que implicaría, asimismo, equilibrio de cuenta corriente. Esto se lograría aplicando a esos modelos la metodología sugerida en la segunda sección de este artículo.

El modelo presentado en el artículo se puede extender de diversas maneras: en la versión de corto-mediano plazo es posible incluir el papel de las expectativas de una forma similar a la que utilizan Sargent y Wallace (1973) o Dornbusch (1976). También es posible incluir diversas formas de rigidez de precios de corto-mediano plazo, especialmente en la versión de la primera sección. Al hacerlo, la política monetaria deja de ser neutral y es posible utilizarla para estabilizar el producto.

Una última conclusión de este trabajo es que, la estructura del modelo keynesiano por el lado de la demanda sigue siendo tal vez la herramienta más útil para explicar los efectos reales de la política fiscal y de los choques externos, al tiempo que es posible lograr que estos modelos observen una estabilidad de largo plazo de la cuenta corriente y la de capitales. Estos resultados son congruentes con la recomendación de Gordon (2009) de volver a estructuras teóricas que parecen tener mayor relación con la realidad que los modelos más modernos. ${ }^{11}$

11 Diversos modelos muy recientes, los cuales surgieron a raíz de la crisis financiera de Estados Unidos de 20082009, analizan el papel de la política fiscal: en este caso están los modelos de Christiano, Eichembaum y Rebelo (2009), Cogan et al. (2010) y Woodford (2010). 


\section{Bibliografía}

Barro, R. (1974), “Are Government Bonds Net Wealth?”, Journal of Political Economy, núm. 82, pp. 1095-1117.

Bernheim, D. (1987), "Ricardian Equivalence: An Evaluation of Theory and Evidence", NBER Macroeconomics Annual 1987, pp. 263-304, National Bureau of Economic Research.

Blanchard, O. (1985), "Debt, Deficits and Finite Horizons", Journal of Political Economy, núm. 93, pp. 223-247.

Blanchard, O. (2008), "The State of Macro", NBER Working Paper 14259, National Bureau of Economic Research.

Blanchard, O. y S. Fischer (1989), Lectures on Macroeconomics, The MIT Press, Cambridge, Massachusetts.

Blanchflower, D. y A. Oswald (1994), The wage curve, MIT Press. Primera edición.

Buiter, W. (2009), "The Unfortunate Uselessness of Most State of the Art Academic Monetary Economics", <http://blogs.ft.com/maverecon/2009/03/ the-unfortunate-uselessness-of-most-state-of-the-art-academic-monetaryeconomics/>.

Calvo, G. (1986), "Temporary stabilization: Predetermined exchange rates”, Journal of Political Economy, núm. 94, pp. 1319-1329.

Christiano, L., M. Eichenbaum y S. Rebelo (2009), "When is the government spending multiplier large?”, NBER Working Paper 15394, National Bureau of Economic Research. Cambridge, Massachusetts.

Cogan, J., T. Cwik y J.B. Taylor (2010), "New Keynesian versus old Keynesian Government Spending Multipliers", Journal of Economics, Dynamics and Control, núm. 34, pp. 281-295.

Dornbusch, R. (1976), "Expectations and exchange rate dynamics”, Journal of Political Economy, núm. 84, pp. 1161-1176.

Dornbusch, R. (1980), Open Economy Macroeconomics, Harper and Row.

Dornbusch, R. y S. Fischer (1978), Macroeconomics, McGraw Hill, Nueva York.

Drazen, A. y E. Helpman (1987), "Stabilization with Exchange Rate Management", Quarterly Journal of Economics, núm. 102, pp. 835-855.

Driffin, J. y M. Miller (2009), "Liquidity Constraints and Non Market Clearing: A Recipe for Recession?", En 6-7th November 2009 CESifo Conference: What is wrong with Modern Macroeconomics?, CEFiso Munich. 
Edwards, S. (2004), "Financial Openness, Sudden Stops and Current Account Reversals", NBER Working Paper 10277, National Bureau of Economic Research.

Funke, K. y C. Nickel (2006), "Does Fiscal Policy Matter for the Trade Account?: A Panel Cointegration Study", Working Paper Series 620, European Central Bank.

Giavazzi, F. and M. Pagano (1996), "Non Keynesian Effects of Fiscal Policy Changes: International Evidence and the Swedish Experience", Swedish Economic Policy Review, núm. 3, pp. 67-103.

Gordon, R. (1978), Macroeconomics, Boston, Little Brown.

Gordon, R. (2009), "Is Modern Macro or 1978-era Macro More Relevant to the Understanding of the Current Economic Crisis?", Artículo presentado en el Coloquio Internacional de Historia del Pensamiento Económico en Sao Paolo, Brasil, Agosto de 2009.

Harrigan, F., P. McGregor, K. Swales y Ya Ping Yin (1993), “Openness, imperfect competition and the NAIRU”, Journal of Economic Studies, núm. 20, pp. 52-72.

Helpman, E. y A. Razin (1987), "Exchange Rate Management: Intertemporal Tradeoffs", American Economic Review, pp. 107-123.

Kim, S. y N. Roubini (2004), “Twin Deficits or Twin Divergence?: Fiscal Policy, the Current Account and Real Exchange Rate in the US", Artículo presentado en el Encuentro de Invierno de la Sociedad Econométrica para Norte América. Mimeo.

Krugman, P. (2009), "How Did Economists Get it So Wrong", New York Times Magazine, September $6^{\text {th }}$, pp. $30 \mathrm{ff}$.

Layard, R. S. Nickell y R. Jackman (1991), Unemployment, Oxford University Press.

Millesi Ferreti, G. y A. Razin (1998), "Current Account Reversals and Currency Crises: Emprical Regularities”, NBER Working Paper 6620, National Bureau of Economic Research.

Mundell, R. (1968), International Economics, McMillan, Nueva York.

Nickel, C. e I. Vansteenkiste (2008), "Fiscal Policies, the Current Account and Ricardian Equivalence", Working Paper Series 935, European Central Bank.

Obstfeld, M. (1985), "The capital inflows problem revisited: A stylized model of the Southern Cone disinflation", Review of Economic Studies, núm. 52, pp. 605623.

Romer, D. (2000), "Keynesian Macroeconomics Without the LM Curve", Journal of Economic Perspectives, núm. 14, pp. 149-169. 
Sargent, T. y N. Wallace (1973), "The Stability of Models of Money and Growth", Econometrica, 41, pp. 1043-1048.

Sutherland, A. (1995), "Fiscal Crises and Aggregate Demand: Can High Public Debt Reverse the Effects of Fiscal Policy?", CEPR Discussion Paper 1246, Center for Economic Policy Research.

Taylor, J. (1993), "Discretion versus policy rules in practice", Carnegie-Rochester Conference Series in Public Policy, núm. 39, pp. 195-214.

Tobin, J. (1993), "Price Flexibility and Output Stability: An Old Keynesian View", Journal of Economic Perspectives, núm. 7, pp. 45-75.

Woodford, M. (2001), "The Taylor Rule and Optimal Monetary Policy”, American Economic Review, núm. 2, pp. 232-237.

Woodford, M. (2008), "Convergence in Macroeconomics: Elements of a New Synthesis", Mimeo.

Woodford, M. (2010), "Simple Analytics of the Government Expenditure Multiplier." Artículo preparado para la Sesión Fiscal Stabilization Policy, Allied Social Science Association, Atlanta, Georgia. 